\title{
Granos secos de destilería con solubles y enzimas en dietas para pollos de engorda
}

\section{Dried Distillers Grains with Solubles and Enzymes in diets for broiler chickens}

\author{
Sergio Gómez-Rosales ${ }^{1,2 *}$ María de Lourdes Ángeles ${ }^{1}$ \\ ${ }^{1}$ Centro Nacional de Investigación Disciplinaria en Fisiología y Mejoramiento Animal. Instituto Nacional de Investigaciones \\ Forestales, Agrícolas y Pecuarias. CP. 76280 Ajuchitlán, Querétaro, México \\ ${ }^{2}$ Facultad de Estudios Superiores Cuautitlán - UNAM. Ajuchitlán, Querétaro, México \\ ${ }^{*}$ Autor de correspondencia: gomez.sergio@inifap.gob.mx
}

Artículo científico recibido: 24 de abril de 2016, aceptado: 23 de noviembre de 2016

RESUMEN. El objetivo del trabajo fue evaluar variables productivas en pollos en crecimiento y finalización alimentados con diferentes concentraciones de granos secos de destilería con solubles (GSDS) y enzimas en dietas con contenido adecuado o baja energía metabolizable (EM), calcio y fósforo para pollos de engorda. En los tres experimentos realizados, los pollos se alojaron en jaulas individuales; en el experimento 1 y 2, 96 y 80 pollos en crecimiento y finalización se asignaron a dietas de sorgo o maíz-pasta de soya en concentraciones de 0, 5, 10 y $15 \%$ de GSDS; en el experimento 3 , 150 pollos se asignaron a dietas con contenido adecuado o bajo en EM, calcio y fósforo y $10 \%$ de GSDS adicionadas con fitasa, glucanasa y xilanasa. Los datos se analizaron por ANOVA, regresión lineal y contrastes ortogonales. En los experimentos 1 y 2 , con análisis de primera derivada, se encontró que para una adecuada productividad en pollos en crecimiento $(\mathrm{p}<0.05)$ y finalización $(\mathrm{p}<0.01)$ el nivel óptimo de GSDS fue de 8.5 y $13 \%$; y para optimizar el peso de la pechuga el nivel máximo fue $4.44 \%$. En el experimento 3, la adición de enzimas compensó la reducción de EM, calcio y fósforo en dietas con $10 \%$ de GSDS. Se puede incluir entre 8.5 y $13 \%$ de GSDS en dietas para pollos en crecimiento y finalización, la adición de enzimas mejora el uso de la EM en dietas con GSDS.

Palabras clave: EM, fitasa, glucanasa, productividad, xilanasa

ABSTRACT. The aim of this study was to evaluate productive variables in growing and finishing chickens fed with different concentrations of dried distillers grains with solubles (DDGS) and enzymes in diets with adequate or low metabolizable energy (ME), calcium and phosphorus content for broilers. In the three experiments conducted, the chickens were housed in individual cages; in experiments 1 and 2, 96 and 80 growing and finishing chickens were assigned to diets of sorghum or corn-soybean paste at concentrations of $0,5,10$ and $15 \%$ DDGS; in experiment 3, 150 chickens were placed on diets with adequate or low ME, calcium and phosphorus content and $10 \%$ DDGS supplemented with phytase, glucanase and xylanase. Data were analyzed by ANOVA, linear regression and orthogonal contrasts. In experiments 1 and 2, with first derivative analysis, it was found that for optimal productivity in growing $(P<0.05)$ and finishing $(P<0.01)$ chickens the optimal DDGS level was 8.5 and $13 \%$, and to optimize the breast weight the maximum level was $4.44 \%$. In experiment 3, the addition of enzymes compensated for the reduction of ME, calcium and phosphorus in diets with $10 \%$ DDGS. Between 8.5 and $13 \%$ DDGS can be included in diets for growing and finishing chickens, and the addition of enzymes improves the use of ME in diets with DDGS.

Key words: ME, phytase, glucanase, productivity, xilanase

\section{INTRODUCCIÓN}

En las últimas décadas se ha observado un incremento en la disponibilidad de los granos secos de destilería con solubles (GSDS) en los Estados Unidos, como consecuencia del acelerado crecimiento de la producción de etanol, lo que ha motivado el interés por estudiar la inclusión de este 
co-producto en la alimentación de las aves (Cortés et al. 2012, Dozier y Hess 2015). En México, el uso de GSDS, derivados del maíz, en la alimentación de pollos de engorda empieza a generalizarse, pero todavía existe preocupación por la industria avícola y fabricantes de alimentos, debido a que no se cuenta con información fidedigna de la composición química de este ingrediente (Olukosi y Adebiyi 2013, Meloche et al. 2014).

Estudios recientes reportan que la composición química de los GSDS, producidos en plantas de nueva generación, es variable, debido a factores como la composición química del grano de maíz, los métodos de procesamiento y la proporción de solubles de destilería agregados a los granos (Loar et al. 2012). Después del proceso de fermentación y secado, la concentración de grasa, proteína, fibra y minerales en los GSDS aumentan en tres veces con respecto a su concentración inicial (Stein y Shurson 2008). El contenido reportado de fósforo disponible, lisina y energía metabolizable (EM) en los GSDS se encuentra entre 0.49 y $0.75 \%, 0.59$ y $0.89 \%$ y, 1869 y $3634 \mathrm{kcal} \mathrm{kg}^{-1}$, respectivamente; lo que refleja una amplia fluctuación de estos nutrientes (Olukosi y Adebiyi 2013, Meloche et al. 2014). Debido a lo anterior, se han observado resultados variables en el comportamiento productivo de los pollos con dietas que contienen GSDS, por lo que normalmente se recomienda incluir un $5 \%$ (Dozier y Hess 2015), o entre 12 y $15 \%$ en pollos en crecimiento y finalización (Loar et al. 2012). En dietas con sorgo y pasta de soya se puede incluir un $6 \%$ para pollos de 1 a 42 d de edad (Cortés et al. 2012).

Una estrategia para tener mejores resultados y lograr mayores niveles de inclusión de GSDS en pollos, es la formulación de dietas isocalóricas, con un balance ideal de AA digestibles, lo que implica conocer la disponibilidad de nutrientes en los GSDS (Shim et al. 2011). Otra estrategia para mejorar el uso digestivo de los GSDS es la inclusión de complejos con actividad multienzimática, para hidrolizar las fracciones de fibra o polisacáridos no-amilaceos (PNA's) de los GSDS (Coppedge et al. 2012, Masey O'Neill et al. 2012). Los PNA's en las paredes celulares de los granos de cereales como el maíz son variados y complejos, siendo los polímeros predominantes los arabinoxilanos, glucanos y celulosa. Los PNA's localizados en el pericarpio del maíz, no son muy abundantes, pero son factores anti nutricionales, que en el intestino forman geles, aumentando la viscosidad y reduciendo la digestibilidad de nutrientes del alimento (Knudsen 2014). Adicionalmente, se ha observado que la adición de fitasa (Martínez-Amezcua et al. 2007) o la combinación de fitasa y enzimas glucosidasas en dietas para pollos puede incrementar la disponibilidad de EM y fósforo (Juanpere et al. 2005). Derivado de lo anterior, el objetivo del trabajo fue evaluar variables productivas en pollos en crecimiento y finalización alimentados con diferentes concentraciones de granos secos de destilería con solubles (GSDS) y enzimas en dietas con contenido adecuado o baja energía metabolizable (EM), calcio y fósforo.

\section{MATERIALES Y MÉTODOS}

El trabajo fue revisado y aprobado por el Comité de Ética de Uso de Animales del Centro Nacional de Investigación Disciplinaria en Fisiología y Mejoramiento Animal del INIFAP; localizado en el kilómetro 1 de la carretera Ajuchitlán-Colón del municipio de Colón, Querétaro, México. En las coordenadas $20^{\circ} 42^{\prime} 18^{\prime \prime} \mathrm{LN}$ y $100^{\circ} 01^{\prime} 09^{\prime \prime} \mathrm{LO}$, a una altitud de $1950 \mathrm{msnm}$. El clima predominante es semi-seco templado con lluvias en verano, con precipitación anual entre 400 y $800 \mathrm{~mm}$, y temperatura de 10 a $20^{\circ} \mathrm{C}$.

El trabajo se formó por tres experimentos de comportamiento productivo con pollos machos de la línea Ross B308, alojados en jaulas individuales elevadas, provistas de comedero de lámina y bebedero de taza, en el interior de una caseta con ventilación natural regulada con cortinas de lona. En los tres experimentos se usó la misma fuente comercial de GSDS, de la cual se obtuvo el coeficiente de digestibilidad ileal aparente (CDIA) de aminoácidos (AA), con excepción de cisteína, triptófano y tirosina; y la energía metabolizable aparente corregida a retención de nitrógeno cero (EMAn) con 
Tabla 1. Contenido total, coeficientes de digestibilidad ileal aparente y aporte de aminoácidos de los granos secos de destilería usados.

\begin{tabular}{lcccccc}
\hline & & & & \multicolumn{3}{c}{ Nivel de GSDS (\%) } \\
\cline { 5 - 7 } Aminoácido (\%) & Totales & CDIA & Digestibles & 5 & 10 & 15 \\
\hline Lisina & 0.873 & 81.80 & 0.71 & 0.04 & 0.07 & 0.11 \\
Metionina & 0.468 & 87.28 & 0.41 & 0.02 & 0.04 & 0.06 \\
Cisteina & 0.783 & 72.00 & 0.56 & 0.03 & 0.06 & 0.08 \\
Treonina & 1.243 & 71.21 & 0.89 & 0.04 & 0.09 & 0.13 \\
Triptofano & 0.269 & 81.00 & 0.22 & 0.01 & 0.02 & 0.03 \\
Arginina & 1.588 & 81.47 & 1.29 & 0.06 & 0.13 & 0.19 \\
Isoleucina & 1.47 & 77.90 & 1.15 & 0.06 & 0.11 & 0.17 \\
Valina & 1.772 & 77.21 & 1.37 & 0.07 & 0.14 & 0.21 \\
Leucina & 2.787 & 72.86 & 2.03 & 0.10 & 0.20 & 0.30 \\
Histidina & 0.965 & 75.28 & 0.73 & 0.04 & 0.07 & 0.11 \\
Fenilalanina & 1.674 & 77.89 & 1.30 & 0.07 & 0.13 & 0.20 \\
\hline
\end{tabular}

el método reportado por Bourdillon et al. (1990). En el Tabla 1 se presenta el contenido total, CDIA y el aporte de AA al sustituir 5, 10 y $15 \%$ de GSDS en el alimento. Para calcular los aportes de cisteína y triptófano se recurrió a los CDIA recomendados por Mariscal et al. (1998). Los CDIA de AA y la EMAn se integraron a la matriz de formulación para ajustar el aporte de AA y energía al incluir los niveles crecientes de GSDS. En el sorgo, maíz y pasta de soya se analizó el contenido de proteína cruda, para calcular el contenido total de AA con las ecuaciones de predicción. Los CDIA de los AA esenciales se calcularon con las tablas publicadas por Mariscal et al. (1998). Las dietas se formularon para cubrir o exceder los requerimientos de nutrientes para pollos en crecimiento y finalización (Rostagno et al. 2005).

\section{Experimento 1}

Se usaron 96 pollos de 21 a 35 d de edad, distribuidos al azar en ocho tratamientos bajo un diseño completamente al azar en arreglo factorial con sorgo o maíz y niveles de GSDS de $0,5,10$ y $15 \%$, con 12 repeticiones por tratamiento. La formulación de las dietas se ajustó para que tuvieran contenidos similares de EM y lisina digestible (Tabla 2). Los aminoácidos se calcularon con base en su digestibilidad ileal verdadera y se mantuvo la proporción de proteína ideal entre los limitantes: lisina, metionina y treonina; el resto de los aminoácidos aportados por los ingredientes fueron excedentes respecto a la relación de proteína ideal.

\section{Experimento 2}

Se usaron 80 pollos de 35 a 49 d de edad, distribuidos al azar en ocho tratamientos bajo un diseño completamente al azar en arreglo factorial con sorgo o maíz, cuatro niveles de GSDS de 0, 5, 10 y $15 \%$, con 10 repeticiones por tratamiento. Todos los procedimientos para la formulación de las dietas fueron similares a los del experimento 1 . La composición calculada de nutrientes de las dietas se presenta en la Tabla 2. Al final del experimento, seis pollos de cada tratamiento se sacrificaron para obtener la pechuga.

\section{Experimento 3}

Se usaron 150 pollos de 32 a 49 d de edad, los cuales fueron distribuidos al azar bajo un diseño completamente al azar en arreglo factorial con sorgo o maíz, con lo que se tuvieron seis tratamientos, los cuales fueron: Dieta CP: control positivo, con pasta de soya como fuente principal de AA y $3.2 \mathrm{Mcal}$ de EM kg ${ }^{-1}$ de alimento; Dieta GSDS: con pasta de soya más $10 \%$ de GSDS como fuentes de AA y 3.2 Mcal de $\mathrm{EM} \mathrm{kg}^{-1}$ de alimento; Dieta GSDS+E: formulada con pasta de soya más $10 \%$ GSDS, pero con reducciones de $0.1 \mathrm{Mcal}$ de $\mathrm{EM} \mathrm{kg}^{-1}$ de alimento y de $0.12 \%$ de Ca y $\mathrm{P}$ disponible. Además se adicionó una combinación de enzimas con actividad de fitasa (200 ppm), $\beta$-glucanasa (175 ppm) y xilanasa $(175 \mathrm{ppm})$. La formulación y composición de las dietas con enzimas se presenta en la Tabla 2, las dietas CP y GSDS corresponden a las dietas con sorgo y maíz del experimento 2 que incluyeron 
Gomez-Rosales y Ángeles

GSDS y enzimas en pollos

Ecosist. Recur. Agropec. 4(11):359-369,2017

Tabla 2. Formulación y contenido calculado de nutrientes de las dietas para pollos en crecimiento y finalización usadas.

\begin{tabular}{|c|c|c|c|c|c|c|c|c|c|c|}
\hline \multirow{3}{*}{$\begin{array}{l}\text { Cereal } \\
\text { Nivel de GSDS, \% } \\
\text { Ingrediente, \% }\end{array}$} & \multicolumn{8}{|c|}{ Experimentos 1 y 2} & \multicolumn{2}{|c|}{ Experimento $3^{a}$} \\
\hline & \multicolumn{4}{|c|}{ Sorgo } & \multicolumn{4}{|c|}{ Maíz } & Sorgo & Maíz \\
\hline & 0 & 5 & 10 & 15 & 0 & 5 & 10 & 15 & 10 & 10 \\
\hline Sorgo molido & $\begin{array}{l}63.6^{*} \\
61.8^{* *}\end{array}$ & $\begin{array}{l}58.9 \\
57.1\end{array}$ & $\begin{array}{l}54.1 \\
52.6\end{array}$ & $\begin{array}{l}49.4 \\
48.2\end{array}$ & 0.00 & 0.00 & 0.00 & 0.00 & 55.37 & 0.00 \\
\hline Maíz molido & 0.00 & 0.00 & 0.00 & 0.00 & $\begin{array}{l}64.2 \\
66.4\end{array}$ & $59.4 / 61.7$ & $54.6 / 56.9$ & $\begin{array}{l}49.6 \\
52.0\end{array}$ & 0.00 & 60.23 \\
\hline Pasta de soya & $\begin{array}{l}28.0 \\
29.4\end{array}$ & $\begin{array}{l}27.4 \\
28.6\end{array}$ & $\begin{array}{l}26.7 \\
27.6\end{array}$ & $\begin{array}{l}26.0 \\
26.0\end{array}$ & $\begin{array}{l}29.1 \\
26.3\end{array}$ & $28.3 / 25.5$ & $27.5 / 24.7$ & $\begin{array}{l}26.7 \\
24.0\end{array}$ & 27.33 & 24.17 \\
\hline GSDS & 0.00 & 5.00 & 10.00 & 15.00 & 0.00 & 5.00 & 10.00 & 15.00 & 10.00 & 10.00 \\
\hline Aceite & $\begin{array}{l}3.58 \\
4.76\end{array}$ & $\begin{array}{l}4.07 \\
5.30\end{array}$ & $\begin{array}{l}4.57 \\
5.80\end{array}$ & $\begin{array}{l}5.06 \\
6.30\end{array}$ & $\begin{array}{l}2.33 \\
3.27\end{array}$ & $2.91 / 3.87$ & $3.50 / 4.50$ & $\begin{array}{l}4.08 \\
5.12\end{array}$ & 3.95 & 2.33 \\
\hline Ortofosfato de calcio & $\begin{array}{l}1.94 \\
1.69\end{array}$ & $\begin{array}{l}1.82 \\
1.55\end{array}$ & $\begin{array}{l}1.70 \\
1.45\end{array}$ & $\begin{array}{l}1.58 \\
1.34\end{array}$ & $\begin{array}{l}1.63 \\
1.40\end{array}$ & $1.53 / 1.31$ & $1.52 / 1.21$ & $\begin{array}{l}1.56 \\
1.12\end{array}$ & 0.89 & 0.63 \\
\hline Carbonato de calcio & $\begin{array}{l}1.31 \\
1.16\end{array}$ & $\begin{array}{l}1.36 \\
1.21\end{array}$ & $\begin{array}{l}1.40 \\
1.25\end{array}$ & $\begin{array}{l}1.45 \\
1.30\end{array}$ & $\begin{array}{l}1.45 \\
1.31\end{array}$ & $1.49 / 1.35$ & $1.53 / 1.38$ & $\begin{array}{l}1.58 \\
1.42\end{array}$ & 1.20 & 1.34 \\
\hline Vitaminas y minerales ${ }^{b}$ & 0.70 & 0.70 & 0.70 & 0.70 & 0.70 & 0.70 & 0.70 & 0.70 & 0.70 & 0.70 \\
\hline Sal & $\begin{array}{l}0.45 \\
0.40\end{array}$ & $\begin{array}{l}0.45 \\
0.40\end{array}$ & $\begin{array}{l}0.45 \\
0.40\end{array}$ & $\begin{array}{l}0.45 \\
0.40\end{array}$ & $\begin{array}{l}0.45 \\
0.40\end{array}$ & $0.45 / 0.40$ & $0.45 / 0.40$ & $\begin{array}{l}0.45 \\
0.40\end{array}$ & 0.40 & 0.40 \\
\hline L-Lisina. $\mathrm{HCl}$ & $\begin{array}{l}0.16 \\
0.00\end{array}$ & $\begin{array}{l}0.17 \\
0.01\end{array}$ & $\begin{array}{l}0.18 \\
0.04\end{array}$ & $\begin{array}{l}0.19 \\
0.06\end{array}$ & $\begin{array}{l}0.09 \\
0.07\end{array}$ & $0.1 / 0.08$ & $0.12 / 0.10$ & $\begin{array}{l}0.14 \\
0.12\end{array}$ & 0.04 & 0.09 \\
\hline DL-Metionina & $\begin{array}{l}0.13 \\
0.11\end{array}$ & $\begin{array}{l}0.13 \\
0.11\end{array}$ & $\begin{array}{l}0.14 \\
0.12\end{array}$ & $\begin{array}{l}0.15 \\
0.13\end{array}$ & $\begin{array}{l}0.10 \\
0.11\end{array}$ & $0.10 / 0.11$ & $0.11 / 0.12$ & $\begin{array}{l}0.12 \\
0.13\end{array}$ & 0.12 & 0.12 \\
\hline L-Treonina & $\begin{array}{l}0.06 \\
0.00\end{array}$ & $\begin{array}{l}0.06 \\
0.00\end{array}$ & $\begin{array}{l}0.06 \\
0.00\end{array}$ & $\begin{array}{l}0.05 \\
0.00\end{array}$ & 0.00 & 0.00 & 0.00 & 0.00 & 0.00 & 0.00 \\
\hline $\begin{array}{l}\text { Fitasa }^{c} \\
\beta \text {-glucanasa }\end{array}$ & $\begin{array}{l}0.00 \\
0.00\end{array}$ & $\begin{array}{l}0.00 \\
0.00\end{array}$ & $\begin{array}{l}0.00 \\
0.00\end{array}$ & $\begin{array}{l}0.00 \\
0.00\end{array}$ & $\begin{array}{l}0.00 \\
0.00\end{array}$ & $\begin{array}{l}0.00 \\
0.00\end{array}$ & $\begin{array}{l}0.00 \\
0.00\end{array}$ & $\begin{array}{l}0.00 \\
0.00\end{array}$ & $\begin{array}{c}0.02 \\
0.018\end{array}$ & $\begin{array}{c}0.02 \\
0.018\end{array}$ \\
\hline $\begin{array}{l}\text { Xilanasa }{ }^{c} \\
\text { Análisis calculado }^{\text {An cal }}\end{array}$ & 0.00 & 0.00 & 0.00 & 0.00 & 0.00 & 0.00 & 0.00 & 0.00 & 0.018 & 0.018 \\
\hline EM, Mcal/kg & $\begin{array}{l}3.10 \\
3.20\end{array}$ & $\begin{array}{l}3.10 \\
3.20\end{array}$ & $\begin{array}{l}3.10 \\
3.20\end{array}$ & $\begin{array}{l}3.10 \\
3.20\end{array}$ & $\begin{array}{l}3.10 \\
3.20\end{array}$ & $3.10 / 3.20$ & $3.10 / 3.20$ & $\begin{array}{l}3.10 \\
3.20\end{array}$ & 3.1 & 3.1 \\
\hline $\mathrm{PC}, \%$ & $\begin{array}{l}18.9 \\
19.3\end{array}$ & $\begin{array}{l}19.5 \\
20.0\end{array}$ & $\begin{array}{l}20.1 \\
20.5\end{array}$ & $\begin{array}{l}20.8 \\
21.0\end{array}$ & $\begin{array}{l}19.4 \\
18.3\end{array}$ & $20.0 / 18.5$ & $20.6 / 19.4$ & $\begin{array}{l}21.2 \\
20.0\end{array}$ & 20.6 & 19.5 \\
\hline Lisina digestible, $\%$ & $\begin{array}{l}1.00 \\
0.91\end{array}$ & $\begin{array}{l}1.00 \\
0.91\end{array}$ & $\begin{array}{l}1.00 \\
0.91\end{array}$ & $\begin{array}{l}1.00 \\
0.91\end{array}$ & $\begin{array}{l}1.00 \\
0.91\end{array}$ & $1.00 / 0.91$ & $1.00 / 0.91$ & $\begin{array}{l}1.00 \\
0.91\end{array}$ & 0.91 & 0.91 \\
\hline Calcio, $\%$ & $\begin{array}{l}0.95 \\
0.85\end{array}$ & $\begin{array}{l}0.95 \\
0.85\end{array}$ & $\begin{array}{l}0.95 \\
0.85\end{array}$ & $\begin{array}{l}0.95 \\
0.85\end{array}$ & $\begin{array}{l}0.95 \\
0.85\end{array}$ & 0.950 .85 & 0.950 .85 & $\begin{array}{l}0.95 \\
0.85\end{array}$ & 0.73 & 0.73 \\
\hline Fósforo disponible, \% & $\begin{array}{l}0.47 \\
0.42\end{array}$ & $\begin{array}{l}0.47 \\
0.42\end{array}$ & $\begin{array}{l}0.47 \\
0.42\end{array}$ & $\begin{array}{l}0.47 \\
0.42\end{array}$ & $\begin{array}{l}0.47 \\
0.42\end{array}$ & 0.470 .42 & 0.470 .42 & $\begin{array}{l}0.47 \\
0.42\end{array}$ & 0.30 & 0.30 \\
\hline
\end{tabular}

*Experimento 1, **experimento 2, ${ }^{a}$ Solo se muestran las dietas que fueron adicionadas con enzimas en el experimento 3 . Las otras dietas usadas corresponden a las dietas con sorgo y maíz del experimento 2 que incluyeron niveles de 0 y $10 \%$ de GSDS, respectivamente, ${ }^{b}$ Cada kg aportó: 6500 UI de vitamina A; 2000 UIP de vitamina D3; 15 Ul de vitamina E; 1.5 mg de vitamina K; 1.5 mg de tiamina; 5 mg de riboflavina; $35 \mathrm{mg}$ de niacina; $3.5 \mathrm{mg}$ de piridoxina; $10 \mathrm{mg}$ de ácido pantoténico; $1500 \mathrm{mg}$ de colina; $0.6 \mathrm{mg}$ de ácido fólico; $0.15 \mathrm{mg}$ de biotina; $0.15 \mathrm{mg}$ de vitamina B12; $100 \mathrm{mg}$ de $\mathrm{Mn} ; 100 \mathrm{mg}$ de Zn; $50 \mathrm{mg}$ de Fe; $10 \mathrm{mg}$ de Cu; $1 \mathrm{mg}$ de I, ${ }^{c}$ Las enzimas fueron provistas por DSM Nutritional Products México. La fitasa (Ronozyme P5000,) es proveniente de Peniophora lycci, que contiene 5000 unidades de fitasa (FTU) por gramo de producto. La $\beta$-glucanasa (Ronozyme VP) es parte de un complejo multienzimático que contiene principalmente actividad de 50 unidades de $\beta$-glucanasa de origen fúngico por gramo, proveniente de Aspergillus aculeatus; también contiene actividad remanente sobre pectinas y hemicelulosa, pero la actividad de estas enzimas no ha sido cuantificada. La xilanasa (Ronozyme WX) es de origen fúngico con una actividad de 1000 unidades de xilanasa por gramo.

niveles de 0 y $10 \%$ de GSDS, respectivamente. Cada tratamiento tuvo 25 repeticiones.

\section{Variables de respuesta}

En todos los experimentos los pollos se pesaron al principio y al final para estimar la ganan- cia diaria de peso (GDP). También se registró el alimento ofrecido y rechazado todos los días, para estimar el consumo diario de alimento (CDA). El consumo diario de EM (CDEM) se obtuvo multiplicando el CDA por el contenido de EM de los alimentos. La conversión alimenticia (CAL) se cal- 
culó dividiendo el consumo de alimento entre la ganancia de peso. La conversión energética (CEN) se estimó dividiendo la ganancia diaria de peso entre el CDEM, y el resultado expresa la cantidad de calorías requeridas para ganar $1 \mathrm{~g}$ de peso. En el experimento 2 se obtuvo el peso de la pechuga en gramos y el rendimiento de la pechuga en porcentaje; este último se calculó dividiendo el peso de la pechuga entre el peso corporal y se multiplicó por 100.

\section{Análisis estadístico}

Los resultados se analizaron con los procedimientos de los modelos lineales generales (SAS 1990). En los experimentos 1 y 2, las diferencias entre medias se evaluaron con el método de la diferencia mínima significativa. Además, se realizaron análisis de regresión lineal simple para conocer el patrón de respuesta de las variables evaluadas con respecto a los niveles de GSDS. Se usó el análisis de la primera derivada, a partir de las ecuaciones de regresión obtenidas para estimar el punto de inflexión de la curva y definir el nivel óptimo de inclusión de GSDS. En el experimento 3 , se usaron contrastes ortogonales para evaluar las diferencias entre medias: Contraste 1 , Sorgo Vs Maíz; contraste 2, CP vs GSDS+E; contraste 3, GSDS vs GSDS+E; Contraste 4, Sorgo (CP vs GSDS+E) vs Maíz (CP vs GSDS+E); contraste 5, Sorgo (GSDS vs GSDS+E) vs Maíz (GSDS vs GSDS+E).

\section{RESULTADOS}

\section{Formulación de las dietas}

El contenido determinado de EMAn fue de 2 $839 \mathrm{kca} \mathrm{kg}^{-1}$ en los GSDS, el cual es menor al estimado para el maíz (3 $390 \mathrm{kcal} \mathrm{kg}^{-1}$ ). Debido a esta diferencia, los cambios más sobresalientes en las dietas fue la reducción de inclusión de sorgo y maíz, entre 7 y $22 \%$, al adicionar de 5 a $15 \%$ de GSDS, mientras que el aceite se incrementó entre 11 y $75 \%$ (Tabla 2). Esto indica que el cambio más sobresaliente de la inclusión de GSDS fue la fuente de energía, ya que se disminuyó el aporte de almidón, y se incrementó la concentración de aceite, ingrediente que provee mayor aporte de energía.

En la Tabla 1 se presenta el contenido de AA totales, el CDIA, AA digestibles y el aporte de AA esenciales al incluir los GSDS entre 5 y $15 \%$ en la dieta. Se observa que el aporte de AA esenciales de los GSDS es bajo, debido a que la pasta de soya se redujo, pero se incrementó la necesidad de AA sintéticos; por ejemplo, la lisina cristalina se incrementó entre 6 y $70 \%$ al aumentar los GSDS de 5 a $15 \%$, mientras que la metionina cristalina estuvo entre 7 y $20 \%$. El contenido de proteína cruda de los GSDS fue de $26.17 \%$, debido a que sustituyeron una mayor proporción en los cereales que en la pasta de soya, la proteína de las dietas aumentó entre 3 y $10 \%$ en los niveles de 5 y $15 \%$ de GSDS. También, se redujo la inclusión de ortofosfato de calcio y se aumentó el carbonato de calcio, debido a que en los GSDS la concentración de fósforo es siete veces mayor.

\section{Experimento 1}

En la Tabla 3 se presentan los resultados de producción y las ecuaciones de regresión en la Tabla 4. La interacción del tipo de cereal y los niveles de GSDS no fueron diferentes estadísticamente para ninguna de las variables evaluadas. Los pollos que recibieron los niveles crecientes de GSDS, tuvieron un patrón cuadrático $(\mathrm{p}<0.05)$ para el CDA, CDEM y GDP, presentando estas tres variables un incremento de 0 a $10 \%$ de GSDS, para luego reducir de forma ligera al $15 \%$ de GSDS. Mientras que la CAL y CEN se redujeron de forma lineal entre 0 y $10 \%$ de GSDS, pero incrementó con $15 \%$ de GSDS con efecto cuadrático $(p<0.05)$. De acuerdo al punto de inflexión de la curva se encontró que el nivel óptimo de inclusión de GSDS fue de $8.25 \%$ para CDA, CDEM y GDP, de $8.88 \%$ para CAL y CEN, y $8.50 \%$ en todas las variables de producción.

\section{Experimento 2}

Los resultados de producción se presentan en el Tabla 3 y las ecuaciones de regresión en la Tabla 4. No se observaron diferencias estadísticas en ninguna de las variables de respuesta con 
Tabla 3. Productividad en pollos en crecimiento (Experimento 1) y finalización (Experimentos 2 y 3 )

\begin{tabular}{|c|c|c|c|c|c|c|c|c|}
\hline & \multicolumn{3}{|c|}{ Cereal } & \multicolumn{5}{|c|}{ GSDS, $\%$} \\
\hline & Sorgo & Maíz & EEMa & 0 & 5 & 10 & 15 & EEMa \\
\hline \multicolumn{9}{|l|}{ Experimento 1} \\
\hline Peso inicial, $\mathrm{g}$ & 576.0 & 583.8 & 14.28 & 572.1 & 590.3 & 583.4 & 573.8 & 20.47 \\
\hline Consumo de alimento, $\mathrm{g} / \mathrm{db}$ & 116.86 & 115.48 & 1.518 & 113.83 & 117.04 & 119.26 & 114.56 & 1.677 \\
\hline Cons. de energía, Kcal/db & 362.28 & 357.99 & 4.705 & 352.88 & 362.81 & 369.72 & 355.14 & 5.748 \\
\hline Ganancia de peso, g/db & 56.06 & 57.10 & 1.309 & 54.34 & 57.01 & 59.06 & 55.90 & 1.377 \\
\hline Conversión alimenticiab & 2.12 & 2.05 & 0.046 & 2.13 & 2.08 & 2.03 & 2.10 & 0.046 \\
\hline Conversión de energíab & 6.59 & 6.35 & 0.142 & 6.61 & 6.46 & 6.30 & 6.50 & 0.153 \\
\hline \multicolumn{9}{|l|}{ Experimento 2} \\
\hline Peso inicial, $\mathrm{g}$ & 2021.2 & 2012.5 & 32.28 & 2031.3 & 2054.5 & 1956.3 & 2025.4 & 51.88 \\
\hline Consumo de alimento, $\mathrm{g} / \mathrm{dcd}$ & 174.29 & 158.74 & 0.771 & 162.91 & 164.83 & 170.15 & 168.16 & 1.073 \\
\hline Cons. de energía, Kcal/dcd & 557.73 & 507.97 & 2.468 & 521.30 & 527.47 & 544.49 & 538.12 & 3.434 \\
\hline Ganancia de peso, g/dd & 75.71 & 77.82 & 1.638 & 69.46 & 74.04 & 82.99 & 80.57 & 2.481 \\
\hline Conversión alimenticiacd & 2.34 & 2.07 & 0.045 & 2.38 & 2.26 & 2.07 & 2.13 & 0.068 \\
\hline $\begin{array}{l}\text { Conversión de energíacd } \\
\text { Pechuga }\end{array}$ & 7.50 & 6.64 & 0.143 & 7.61 & 7.24 & 6.61 & 6.82 & 0.199 \\
\hline Peso, gd & 749.88 & 724.63 & 15.321 & 742.38 & 740.13 & 743.25 & 723.25 & 22.291 \\
\hline Rendimiento, \%e & 24.30 & 23.36 & 0.502 & 24.52 & 24.09 & 23.70 & 23.01 & 0.745 \\
\hline \multicolumn{9}{|l|}{ Experimento 3} \\
\hline & \multicolumn{3}{|c|}{ Cereal } & \multicolumn{5}{|c|}{ Dieta } \\
\hline & Sorgo & Maíz & EEM & CP & GSDS & GSDS+E & EEM & \\
\hline Peso inicial, $g$ & 1243.8 & 1214.4 & 14.37 & 1223.4 & 1226.1 & 1242.1 & 19.66 & \\
\hline Consumo de alimento, $\mathrm{g} / \mathrm{d}$ & 145.17 & 144.43 & 0.536 & 145.03 & 145.07 & 144.74 & 0.741 & \\
\hline Cons. de energía, Kcal/d & 459.85 & 457.97 & 1.676 & $464.05 f$ & $464.21 \mathrm{f}$ & $448.69 \mathrm{~g}$ & 2.322 & \\
\hline Ganancia de peso, g/d & 69.16 & 70.31 & 0.795 & 70.57 & 70.39 & 69.24 & 1.101 & \\
\hline Conversión alimenticia & 2.11 & 2.07 & 0.022 & 2.09 & 2.08 & 2.09 & 0.031 & \\
\hline Conversión de energía & 6.65 & 6.57 & 0.071 & $6.70 \mathrm{~h}$ & $6.65 \mathrm{hi}$ & $6.48 \mathrm{i}$ & 0.097 & \\
\hline
\end{tabular}

Tabla 4. Ecuaciones de regresión, punto de inflexión y coeficiente de regresión de las variables de respuesta obtenidas en los experimentos 1 y 2 .

\begin{tabular}{llrr}
\hline Variable de respuesta & Ecuación de regresión & Punto de inflexióna & $\mathrm{R}^{2}$ \\
\hline Experimento 1 & & & \\
Consumo de alimento g/d & $\mathrm{y}=113.53+140.75 \mathrm{x}-155.89 \mathrm{x}^{2}$ & 8.06 & 0.90 \\
Consumo de energía, kcal/d & $\mathrm{y}=351.95+3.9513 \mathrm{x}-0.2452 \mathrm{x}^{2}$ & 8.06 & 0.90 \\
Ganancia de peso, g/d & $\mathrm{y}=54.12+1.0095 \mathrm{x}-0.0584 \mathrm{x}^{2}$ & 8.64 & 0.91 \\
Conversión alimenticia & $\mathrm{y}=2.1368+0.0198 \mathrm{x}-0.0011 \mathrm{x}^{2}$ & 9.00 & 0.86 \\
Conversión energética & $\mathrm{y}=6.624+0.0613 \mathrm{x}-155.89 \mathrm{x}^{2}$ & 8.76 & 0.86 \\
Experimento 2 & & & \\
Consumo de alimento g/d & $\mathrm{y}=162.37+1.0094 \mathrm{x}-0.0392 \mathrm{x}^{2}$ & 12.88 & 0.82 \\
Consumo de energía, kcal/d & $\mathrm{y}=519.59+3.2302 \mathrm{x}-0.1254 \mathrm{x}^{2}$ & 12.88 & 0.82 \\
Ganancia de peso, g/d & $\mathrm{y}=68.68+1.8937 \mathrm{x}-0.0699 \mathrm{x}^{2}$ & 13.55 & 0.89 \\
Conversión alimenticia & $\mathrm{y}=2.3964-0.0463 \mathrm{x}-0.0018 \mathrm{x}^{2}$ & 12.86 & 0.90 \\
Conversión energética & $\mathrm{y}=7.6685-0.148 \mathrm{x}-0.0058 \mathrm{x}^{2}$ & 12.76 & 0.90 \\
Peso de la pechuga, g & $\mathrm{y}=740.95+1.5775 \mathrm{x}-0.1775 \mathrm{x}^{2}$ & 4.44 & 0.85 \\
\hline Mediante análisis de la primera derivada para estimar el nivel óptimo de inclusión de GSDS.
\end{tabular}

respecto a la interacción de tipo de cereal y nivel de GSDS. Para el tipo de cereal se encontró que el CDA, CDEM, CAL y CEN fueron menores ( $p<$ 0.01 ) en los pollos alimentados con sorgo, comparados con los que recibieron la dieta con maíz. Con la inclusión de GSDS, se encontró que el CDA, CDEM y GDP mostraron un patrón cuadrático $(\mathrm{p}<0.01)$, con incrementos de 0 a $10 \%$ de GSDS, y se redujo de forma ligera con $15 \%$ de GSDS. También la CAL y la CEN se comportaron de forma cuadrática $(p<0.01)$, ya que se redujo de forma lineal entre los niveles de 0 a $10 \%$, y se incrementó con 15 
$\%$ de GSDS. El peso de la pechuga se mantuvo constante entre 0 y $10 \%$ de GSDS, y se redujo al $15 \%$ de GSDS con efecto cuadrático ( $p<0.05$ ). El rendimiento de la pechuga se redujo de forma lineal $(p<0.01)$ al incrementar de 0 a $15 \%$ de GSDS. El nivel óptimo de inclusión de GSDS fue de $13.10 \%$ para CDA, CDEM y GDP, de $12.81 \%$ para CAL y CEN, y de $12.96 \%$ en todas las variables de producción. Para optimizar el peso de la pechuga el nivel máximo de inclusión fue de 4.44 \% (Tabla 4).

\section{Experimento 3}

El CDA, GDP y CAL (Tabla 3) fueron similares entre los pollos que consumieron las dietas $C P$, GSDS y GSDS+E. El CEM fue menor $(p<0.01)$ en los pollos que consumieron la dieta GSDS+E comparados con los que recibieron las dietas $\mathrm{CP}$ y GSDS. La CEN fue menor $(p<0.09)$ en los del grupo GSDS+E, con respecto a los pollos del grupo $\mathrm{CP}$, mientras que en los pollos GSDS la CEN fue intermedia.

\section{DISCUSIÓN}

La fuente evaluada de GSDS contenía 0.71, 0.41 y $0.89 \%$ de lisina, metionina y treonina digestibles (Tabla 1), valores que están dentro del rango de valores reportados en estudios previos (Batal y Dale 2006). En otros estudios se han usado fuentes de GSDS con mayor contenido de AA indispensables totales y digestibles (Wang et al. 2007, Olukosi y Adebiyi 2013). Mientras que Cortés et al. (2012), estimó el contenido de AA digestibles en dos fuentes de GSDS por espectroscopia de reflectancia infrarroja, reportando valores de lisina, metionina y treonina superiores a los de los GSDS del presente trabajo. El contenido de EMAn de la muestra de GSDS usada en el presente trabajo se encuentra dentro del rango reportado por Adeola y Zhai (2012) y Meloche et al. (2014).

Lo encontrado en los experimentos 1 y 2 indica que las dietas formuladas con maíz o sorgo, el nivel óptimo de inclusión de GSDS es de 8.5 y $13 \%$, respectivamente. Para GSDS de nueva generación, se recomienda incluir de 5 a $15 \%$ para pollos en crecimiento y finalización (Lumpking et al. 2004, Loar et al. 2012, Dozier y Hess 2015). Mientras que Waldroup et al. (1981) reportaron que cuando los GSDS son incluidos en dietas isocalóricas para pollos, se puede incluir hasta $25 \%$, sin tener efectos negativos en el peso corporal o conversión alimenticia. En tanto que Wang et al. (2007) con niveles de 0 a $25 \%$ de GSDS en pollos de 1 a 49 d, con una matriz de nutrientes estandarizada, encontraron que puede incluirse GSDS en niveles de 15 a $20 \%$ en la dieta, con mínimos efectos negativos sobre el comportamiento productivo, reducción del rendimiento de la canal y la pechuga.

Al igual que en el presente estudio, Loar et al. (2012) determinaron la digestibilidad de AA y el contenido de energía en una muestra de GSDS, encontrando que al aumentar el nivel de GSDS de 0 a $8 \%$ en las dietas de iniciación y crecimiento, se incrementó la conversión alimenticia y se redujo la ganancia de peso, mientras que en la etapa de finalización, con contenidos mayores de $14 \%$ de GSDS, observaron disminución en el consumo de alimento y ganancia de peso; lo que coincide con lo encontrado en el presente estudio. Mientras que Shim et al. (2011) al incluir niveles de 0 a $24 \%$ de GSDS con niveles estables de AA digestibles, observaron que, en la fase de iniciación y finalización, los pollos con dietas con niveles de GSDS de 8 a $24 \%$ tuvieron mayor peso corporal, pero el consumo del alimento y conversión alimenticia no fue diferente entre tratamientos. Los mayores pesos en pollos que consumieron entre 8 y $24 \%$ de GSDS coinciden con los efectos cuadráticos observados en la productividad de los pollos en crecimiento y finalización del presente trabajo. Mientras que Cortés et al. (2012) reportaron que en pollos de engorda alimentados con dietas basadas en sorgo-pasta de soya con GSDS de 0 a $21 \%$, la ganancia de peso se redujo, mientras que el consumo de alimento y la conversión alimenticia se incrementó. Al respecto Cortés et al. (2012) recomiendan la inclusión de un máximo de $7 \%$ de GSDS en los alimentos de pollos de 1 a $49 \mathrm{~d}$ de edad, el cual es un nivel inferior a los niveles óptimos de 8.5 y $13 \%$ de GSDS para pollos en crecimiento y finalización encontrados en el presente trabajo. 
El peso de la pechuga se redujo con la inclusión de $15 \%$ de GSDS (Tablas 3 y 4), mientras que el rendimiento se redujo de forma lineal con el incremento de GSDS. El grado de reducción del rendimiento de la pechuga fue de $1.8,3.4$ y $6.2 \%$ con 5,10 y $15 \%$ de GSDS, respectivamente. Al respecto Jung et al. (2012) encontraron que la inclusión de 6 y $12 \%$ de GSDS en la dieta de pollos, no afecta el peso de la canal y la pechuga, pero reduce el rendimiento de la canal. En tanto que Dozier y Hess (2015) con niveles de 0 a $12 \%$ de GSDS, no detectaron afectos negativos en el peso de la canal y la pechuga; pero el rendimiento de la canal se redujo cuando se incrementó la GSDS con alto contenido de aceite. También se han reportado reducciones en el peso o rendimiento de la canal y la pechuga en pollos con dietas de 14 a $25 \%$ de GSDS (Loar et al. 2012, Wang et al. 2007). Mientras que Lumpking et al. (2004) y Cortés et al. (2012) reportan efectos negativos en el peso y rendimiento de la canal y la pechuga en pollos alimentados con dietas de 18 , 21 y $24 \%$ de GSDS. Otros efectos son la reducción de la grasa abdominal (Shim et al. 2011, Dozier y Hess 2015) y el incremento en el peso relativo de la molleja, intestino delgado e intestino grueso (Loar et al. 2012). Lo que sugiere que el contenido de fibra de los GSDS provoca un incremento de la masa visceral, lo que aumenta el requerimiento de nutrientes para el mantenimiento y crecimiento de los epitelios digestivos, específicamente de energía y AA, desviando estos nutrientes de la síntesis de proteína del músculo de la pechuga. Por lo que, si se quiere maximizar el peso de la pechuga, se recomienda incluir niveles de GSDS menores del 4.44 $\%$ en la dieta de pollos en finalización.

En el experimento 3 (Tabla 3), la reducción en el CEM en los pollos que consumieron la dieta GSDS+E, baja en nutrientes con enzimas, se observo menor conversión energética, debido a que la actividad de las enzimas adicionadas tiene efecto en la hidrolización de los PNA's de las dietas, los cuales pueden convertirse en factores antinutricionales (Knudsen 2014). La productividad fue similar entre los pollos que consumieron las tres dietas, lo que indica que se puede incluir hasta $13 \%$ de GSDS en pollos en finalización sin efectos negativos en la producción. La conversión energética fue estadísticamente diferente entre el CP y GSDS+E, debido a que las calorías requeridas para ganar un gramo de peso fue menor en la segunda dieta; esto corrobora la mejora en la conversión alimenticia observada en los pollos adicionados con niveles crecientes de GSDS (Tablas 3 y 4 ) e indica que la menor conversión alimenticia se logró con $13 \%$ de GSDS.

La falta de diferencias estadísticas en la ganancia de peso, consumo de alimento y conversión alimenticia entre la dieta CP y GSDS con la GSDS+E coincide con Cowieson et al. (2010) quienes encontraron que pollos con una dieta de $0.10 \mathrm{Mcal}$ de $\mathrm{EM} \mathrm{kg}^{-1}$ formulada con maíz y adicionada con xilanasa, tuvieron la misma conversión alimenticia que los pollos alimentados con dieta CP, pero mayor conversión alimenticia que los pollos alimentados con la dieta CN sin xilanasa. Mientras que Campasino et al. (2015) reportan que en pollos de 1 a $48 \mathrm{~d}$ de edad alimentados con una dieta $\mathrm{CN}$, baja en energía con la adición de xilanasa, glucanasa y galactosidasa el peso corporal, peso de la canal y componentes de la canal fueron similares entre los pollos que consumieron la dieta CN y la dieta CP. Resultados similares fueron observados por Coppedge et al. (2012) y Masey O'Neill et al. (2012) sobre el peso corporal y conversión alimenticia de pollos alimentados con dietas bajas en energía suplementadas con enzimas.

En las dietas con maíz con la inclusión de xilanasas y glucanasas, se han reportado mejoras en la producción, digestibilidad ileal de la energía y EM (Massey O'Neill et al. 2012). Mientras que en dietas con sorgo adicionadas con xilanasa, glucanasa y proteasa se observó incremento en el peso corporal y la conversión alimenticia (Selle et al. 2010). Mientras que en dietas que incluyeron de 0 a $20 \%$ de GSDS de maíz, la adición de xilanasa aumentó el peso, digestibilidad total e ileal de la materia seca, proteína cruda, energía y hemicelulosa, pero redujo la conversión alimenticia (Liu et al. 2011). Con GSDS de sorgo, se ha encontrado que la combinación de proteasa y xilanasa mejora la productividad cuando se incluyó 20 \% de GSDS en la dieta 
(Barekatain et al. 2013). Lo que indica que estas enzimas son adecuadas para hidrolizar las paredes celulares de los granos o subproductos de estos, liberando mayor cantidad de nutrientes.

La inclusión de fitasa en dietas con maíz o sorgo, sin o con la inclusión de GSDS, ha incrementado la digestibilidad y disponibilidad de fósforo (Martínez-Amezcua et al. 2007). También se ha encontrado que la fitasa mejora la digestibilidad ileal de la materia seca, energía y la EM de los alimentos (Juanpere et al. 2005, Leslie et al. 2007). La inclusión de fitasa se utilizó para mejorar la liberación de fósforo y calcio de las dietas. La falta de diferencias en la productividad en los pollos alimentados con la dieta GSDS+E comparada con las dietas CP, sugiere que la fitasa fue capaz de liberar al menos $0.12 \%$ de fósforo y calcio. Sin embargo, no se descarta la posibilidad que la fitasa también haya afectado de manera positiva la utilización de la energía de la dieta.

En los tres experimentos se observó que la conversión alimenticia y conversión energética fueron mayores con sorgo, aunque estas diferencias solo fueron significativas en los pollos en finalización del experimento 2. Las dietas con sorgo se asocian con respuestas productivas inconsistentes $y$, menores con respecto a las dietas formuladas con maíz, debido a la presencia de taninos condensados y proteínas estructurales de baja solubilidad que pueden reducir la digestibilidad de nutrientes en el tracto digestivo (Gómez et al. 2009). Para los pollos en crecimiento y finalización, el nivel óptimo de inclusión de GSDS fue de 8.5 y 13 \% para maíz y sorgo, respectivamente. Al respecto Cortés et al. (2012) encontraron que en dietas con sorgo, el nivel máximo de inclusión de GSDS en pollos de 1 a $49 \mathrm{~d}$ de edad fue del $7 \%$. Probablemente, los niveles máximos de inclusión de GSDS para pollos en iniciación, crecimiento y finalización sean de 7.0, 8.5 y $13 \%$, respectivamente; siempre y cuando se conozca el aporte de EM y de AA digestibles en los GSDS usados y se tomen en cuenta para la formulación de las dietas.

\section{CONCLUSIONES}

Para pollos de engorda en crecimiento y finalización se tuvo un adecuado crecimiento con la inclusión de 8.5 a $13 \%$ de GSDS, respectivamente. Para optimizar el peso de la pechuga, se sugiere usar un nivel máximo de $4.44 \%$ de GSDS en las dietas de finalización. Es importante conocer el aporte de EM y de AA digestibles de los GSDS para obtener un buen balance de nutrientes de la dieta y maximizar los resultados de productividad. En pollos en finalización, la adición de enzimas como fitasa, $\beta$ glucanasa y xilanasa en dietas bajas en EM, calcio y fósforo formuladas con $10 \%$ de GSDS, se puede obtener parámetros productivos similares a los observados en pollos que consumen dietas con niveles recomendados de EM, calcio y fósforo.

\section{LITERATURA CITADA}

Adeola O, Zhai H (2012) Metabolizable energy value of dried corn distillers grains and corn distillers grains with solubles for 6-week-old broiler chickens. Poultry Science 91: 712-718.

Barekatain MR, Antipatis C, Rodgers N, Walkden-Brown SW, lji PA, Choct M (2013) Evaluation of high dietary inclusion of distillers dried grains with solubles and supplementation of protease and xylanase in the diets of broiler chickens under necrotic enteritis challenge. Poultry Science 92: 1579-1594.

Batal AB, Dale NM (2006) True metabolizable energy and amino acid digestibility of distillers dried grains with solubles. Journal of Applied Poultry Research 15: 89-93.

Bourdillon A, Carré B, Conan L, Duperray J, Huyghebaert G, Leclercq B, et al. (1990) European reference method for the in vivo determination of metabolisable energy with adult cockerels: reproducibility, effect of food intake and comparison with individual laboratory methods. British Poultry Science 31: 557-565. 
Campasino A, Williams M, Latham R, Bailey CA, Brown B, Lee JT (2015) Effects of increasing dried distillersgrains with solubles and non-starch polysaccharide degrading enzyme inclusion on growth performance and energy digestibility in broilers. Journal Applied Poultry Research 24: 135-144.

Cortés CA, Esparza CCA, Sanabria EG, Iriarte JM, Ornelas RM, Ávila GE (2012) El uso de granos secos de destilería con solubles (GSDS) en dietas sorgo-soya para pollos de engorda y gallinas de postura. Revista Mexicana de Ciencias Pecuarias 3: 331-341.

Coppedge JR, Oden LA, Ratliff B, Brown B, Ruch F, Lee JT (2012) Evaluation of nonstarch polysaccharidedegrading enzymes in broiler diets varying in nutrient and energy levels as measure by broiler performance and processing parameters. Journal of Applied Poultry Research 21: 226-234.

Cowieson AJ, Bedford MR, Ravindran V (2010) Interactions between xylanase and glucanase in maize soybased diets for broilers. British Poultry Science 51: 246-257.

Dozier III WA, Hess JB (2015) Growth and meat yield responses of Hubbard x Cobb 500 male broilers fed diets formulated with distillers dried grains with solubles varying in ether extract content and inclusion rate from 1 to 33 days of age. Journal of Applied Poultry Research 24: 436-450.

Gómez RS, Angeles ML, Mariscal LG, Mejía GCA, Braña VD, Rentería FJA, et al. (2009) Estrategias para el uso eficiente de materias primas en porcicultura. Centro Nacional de Investigación Disciplinaria en Fisiología y Mejoramiento Animal, INIFAP-SAGARPA. Libro Técnico No. 1, Colón, Querétaro. 54p.

Juanpere J, Pérez-Vendrell AM, Angulo E, Brufau J (2005) Assessment of potential interactions between phytase and glycosidase enzyme supplementation on nutrient digestibility in broilers. Poultry Science 84: 571-580.

Jung B, Mitchell RD, Batal AB (2012) Evaluation of the use of feeding distillers dried grains with solubles in combination with canola meal on broiler performance and carcass characteristics. Journal of Applied Poultry Research 21: 776-787.

Knudsen KEB (2014) Fiber and nonstarch polysaccharide content and variationin common crops used in broiler diets. Poultry Science 93: 2380-2393.

Leslie MA, Moran Jr ET, Bedford MR (2007) The effect of phytase and glucanase on the ileal digestible energy of corn and soybean meal fed to broilers. Poultry Science 86: 2350-2357.

Liu N, Ru YJ, Tang DF, Xu TS, Partridge GG (2011) Effects of corn distillers dried grains with solubles and xylanase on growth performance and digestibility of diet components in broilers. Animal Feed Science and Technology 163: 260-266.

Loar II RE, Donaldson JR, Corzo A (2012) Effects of feeding distillers dried grains with solubles to broilers from 0 to 42 days posthatch on broiler performance, carcass characteristics, and selected intestinal characteristics. Journal of Applied Poultry Research 21: 48-62.

Lumpkins BS, Batal AB, Dale NM (2004) Evaluation of distillers dried grains with solubles as a feed ingredient for broilers. Poultry Science 83: 1891-1896.

Mariscal LG, Ávila E, Tejada HI, Cuarón IJA, Vázquez PC. (1998). Tablas del contenido de aminoácidos totales y de los coeficientes de digestibilidad verdadera para aves y cerdos. INIFAP-PRODUCE. México, DF. 1p.

Martinez-Amezcua C, Parsons CM, Singh V, Srinivasan R, Murthy GS (2007) Nutritional characteristics of corn distillers dried grains with soluble as affected by the amounts of grains versus soluble and different processing techniques. Poultry Science 86: 2624-2630. 
Masey O'Neill HV, Liu N, Wang JP, Diallo A, Hill S (2012) Effect of xylanase on performance and apparent metabolisable energy in starter broilers fed diets containing one maize variety harvested in different regions of China. Asian-Australasian Journal Animal Science 25: 515 - 523.

Meloche KJ, Kerr BJ, Billor N, Shurson GC, Dozier III WA (2014) Validation of prediction equations for apparent metabolizable energy of corn distillers dried grains with solubles in broiler chicks. Poultry Science 93: 1428-1439.

Olukosi OA, Adebiyi AO (2013) Chemical composition and prediction of amino acid content of maize- and wheat-distillers? dried grains with soluble. Animal Feed Science and Technology 185: 182- 189.

Rostagno HS, Albino LFT, Donzelle JL, Gomes PC, Oliveira RF, Lopes DC, et al. (2005) Brazilian Tables for Poultry and Swine. Composition of Feedstuffs and Nutritional Requirements. 2nd ed. Vicosa: UFV, Departamento de Zootecnia 95-113.

Selle PH, Cadogan DJ, Ru YJ, Partridge GG (2010) Impact of exogenous enzymes in sorghum- or wheatbased broiler diets on nutrient utilization and growth performance. International Journal of Poultry Science 9: 53-58.

SAS (1990) SAS user's guide: Statistics. Version 6 (4th ed). Statistical Analysis Systems Institute, Inc, Cary, NC, USA.

Shim MY, Pesti GM, Bakalli RI, Tillman PB, Payne RL (2011) Evaluation of corn distillers dried grains with solubles as an alternative ingredient for broilers. Poultry Science 90: 369-376.

Stein HH, Shurson GC (2008) Board-invited review: The use and application of distillers dried grains with solubles (DDGS) in swine diets. Journal of Animal Science 87: 1292-1303.

Waldroup PW, Owen JA, Ramsey BE, Whelchel DL (1981) The use of high levels of distillers dried grains plus solubles in broiler diets. Poultry Science 60: 1479-1484.

Wang Z, Cerrate S, Coto C, Yan F, Waldroup PW (2007) Utilization of distillers dried grains with solubles (DDGS) in broiler diets using a standardized nutrient matrix. International Journal of Poultry Science 6: 470-477. 
